\title{
Scalarization of slowly rotating black holes
}

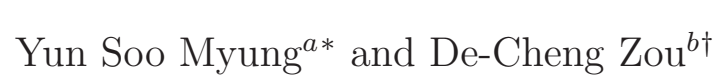 \\ ${ }^{a}$ Institute of Basic Sciences and Department of Computer Simulation, Inje University \\ Gimhae 50834, Korea \\ ${ }^{b}$ Center for Gravitation and Cosmology and College of Physical Science and Technology, \\ Yangzhou University, Yangzhou 225009, China
}

\begin{abstract}
It is interesting to note that most black holes are born very slowly rotating. We investigate scalarization of slowly rotating black holes in the Einstein-scalar-ChernSimons (EsCS) theory. In the slow rotation approximation, the CS term takes a linear form of rotation parameter $a$ which determines the tachyonic instability. The tachyonic instability for slowly rotating black holes represents the onset of spontaneous scalarization. It is shown that the slowly rotating black holes are unstable against a spherically symmetric scalar-mode perturbation for positive coupling $\alpha$, whereas these black holes are unstable for negative coupling without any $a$-bound.
\end{abstract}

*e-mail address: ysmyung@inje.ac.kr

†e-mail address: dczou@yzu.edu.cn 


\section{Introduction}

Scalarization of rotating (Kerr) black holes has been investigated by making use of the Einstein-scalar-Gauss-Bonnet (EsGB) theory with positive scalar coupling parameter [1, 2]. The tachyonic instability for rotating black holes is regarded as the onset of spontaneous scalarization. In these works, it was noted that the sufficiently high rotations $(a \geq 0.5)$ suppresses scalarization because the GB term is not monotonic and could become negative around the outer horizon.

Recently, the onset of scalarization for Kerr black holes was found as an $a$-bound of $a / M \geq 0.5$ (sufficiently high rotations) in the EsGB theory with negative coupling parameter [3]. This a-bound was confirmed analytically by considering an asymptotic $(l \rightarrow \infty)$-scalar mode [4] and numerically by considering the $(2+1)$-dimensional evolution equation [5]. Also, it is shown that any instability could be not triggered for $a<a_{\text {min }}=0.5$ with $M=1$ in the EsGB theory [6]. These imply that the threshold of instability depends on both the coupling and rotation parameters. As results, the spin-induced scalarized black holes were numerically constructed for $a / M \geq 0.5$ in the EsGB theory with negative coupling parameter [7, 8]. Here, we wish to point out that most of spin-induced black hole scalarizations were realized through scalar coupling to the GB term.

On the other hand, we have found the $a>a\left(\alpha_{\mathrm{th}}\right)$-bound without an $a$-bound when studying the instability of rotating black holes in the Einstein-scalar-Chern-Simons (EsCS) theory with negative coupling parameter [9]. For positive coupling in the EsCS theory, the tachyonic instability for Kerr black hole were investigated firstly in [10] and its scalarized rotating black holes were constructed [11]. More recently, the tachyonic instability for Kerr black hole were discussed for a massive scalar in the EsCS theory [12].

Now, it is interesting to introduce a slowly rotating black hole because it intrinsically forbids sufficiently high rotations $(a \geq 0.5)$. This black hole could be found when keeping all quantities of interest up to first order in $a$ (that is, $a \ll 1$ ). At this stage, we would like to mention that most black holes are born very slowly rotating [13]. For example, black holes born from single stars rotate very slowly with $a=0.01$ and fairly slow rotating black holes born from single stars are the cases with $a \leq 0.1$. Hence, studying slowly rotating black holes will reveal how $a$ plays the role in achieving spontaneous scalarization for positive and negative coupling parameter $\alpha$.

In this work, we wish to study the onset of scalarization for slowly rotating black holes in 
the EsCS theory with the coupling parameter $\alpha$. Its linearized scalar equation includes the CS term depending on $a \cos \theta$. This may imply no $a$-bound for spontaneous scalarization for negative coupling $\alpha$. If one considers the linearized scalar theory around slowly rotating black holes in the EsGB theory, it involves $48 M^{2} / r^{6}$ which is independent of $a \cos \theta$, nothing to do with rotation. This is the reason why we consider the EsCS theory for investigating scalarization of slowly rotating black holes. We will employ the $(2+1)$-dimensional hyperboloidal foliation method to show the tachyonic instability of slowly rotating black hole by considering the time evolution of a spherically symmetric scalar-mode.

\section{Slowly rotating black holes}

The EsCS theory takes the form

$$
S=\frac{1}{16 \pi} \int d^{4} x \sqrt{-g}\left[R-\frac{1}{2}(\partial \phi)^{2}+\alpha \phi^{2 *} R R\right]
$$

with geometric units of $G=c=1$. In our model (1), we choose the quadratic coupling function to the CS term

$$
{ }^{*} R R={ }^{*} R_{\xi}^{\eta \mu \nu} R_{\eta \mu \nu}^{\xi} .
$$

Here the dual Ricci tensor is defined as ${ }^{*} R_{\xi}^{\eta \mu \nu}=\frac{1}{2} \epsilon^{\mu \nu \rho \sigma} R_{\xi \rho \sigma}^{\eta}$ with the Levi-Civita tensor $\epsilon^{\mu \nu \rho \sigma}$. Varying (11) with respect to $g_{\mu \nu}$ and $\phi$ leads to Einstein and scalar equations as

$$
\begin{aligned}
& G_{\mu \nu}=\frac{1}{2} \partial_{\mu} \phi \partial_{\nu} \phi-\frac{1}{4} g_{\mu \nu}(\partial \phi)^{2}-4 \alpha C_{\mu \nu} \\
& \nabla^{2} \phi+2 \alpha^{*} R R \phi=0
\end{aligned}
$$

where $C_{\mu \nu}$ the Cotton tensor is determined by

$$
C_{\mu \nu}=\nabla_{\rho}\left(\phi^{2}\right) \epsilon_{(\mu}^{\rho \sigma \gamma} \nabla_{\gamma} R_{\nu) \sigma}+\frac{1}{2} \nabla_{\rho} \nabla_{\sigma}\left(\phi^{2}\right) \epsilon_{(\nu}^{\rho \gamma \delta} R_{\mu) \gamma \delta}^{\sigma} .
$$

Considering $\phi=0$, Eq. (3) reduces to $R_{\mu \nu}=0$ which implies the Kerr spacetime written in Boyer-Lindquist coordinates $\{t, r, \theta, \varphi\}$

$$
\begin{aligned}
d s_{\text {Kerr }}^{2} & \equiv \tilde{g}_{\mu \nu} d x^{\mu} d x^{\nu} \\
& =-\frac{\Delta}{\rho^{2}}\left(d t-a \sin ^{2} \theta d \varphi\right)^{2}+\frac{\rho^{2}}{\Delta} d r^{2}+\rho^{2} d \theta^{2}+\frac{\sin ^{2} \theta}{\rho^{2}}\left[a d t-\left(r^{2}+a^{2}\right) d \varphi\right]^{2}
\end{aligned}
$$


with mass $(M)$, angular momentum $(J)$, rotation parameter $(a=J / M>0), \Delta=r^{2}-$ $2 M r+a^{2}$, and $\rho^{2}=r^{2}+a^{2} \cos ^{2} \theta$. It is worth noting that Eq. (6) describes a stationary, axisymmetric, and non-static spacetime. In this case, taking into account $\Delta=0$ leads to the outer and inner horizons as

$$
\tilde{r}_{ \pm}=M\left[1 \pm \sqrt{1-\frac{a^{2}}{M^{2}}}\right] .
$$

Before we proceed, we mention the CS term based on Eq. (6). Its form is given by

$$
\begin{aligned}
* \tilde{R} \tilde{R} & =\frac{96 r M^{2} a \cos \theta\left(3 r^{4}-10 r^{2} a^{2} \cos ^{2} \theta+3 a^{4} \cos ^{4} \theta\right)}{\rho^{12}} \\
& \simeq \frac{96 M^{2} a \cos \theta}{r^{7}}\left[3-\frac{28 a^{2} \cos ^{2} \theta}{r^{2}}+\cdots\right],
\end{aligned}
$$

where ${ }^{*} \tilde{R} \tilde{R}$ is odd with respect to parity transformation: ${ }^{*} \tilde{R} \tilde{R}(\pi-\theta)=-{ }^{*} \tilde{R} \tilde{R}$. This parity property plays an important role in deriving the threshold curves for negative coupling $\alpha$ when combining with the transformation of $\alpha \rightarrow-\alpha$.

From now on, we consider the slowly rotating black hole keeping up to $\mathcal{O}(a)$-order in the slow rotation approximation $a \ll 1(J \ll M)$ [14, 15]

$$
\begin{aligned}
d s_{\mathrm{SR}}^{2} & =\bar{g}_{\mu \nu} d x^{\mu} d x^{\nu} \\
& =-\left(1-\frac{2 M}{r}\right) d t^{2}+\frac{d r^{2}}{1-\frac{2 M}{r}}+r^{2}\left(d \theta^{2}+\sin ^{2} \theta d \varphi^{2}\right)+\frac{4 a M \sin ^{2} \theta}{r} d t d \varphi
\end{aligned}
$$

which is still a stationary, axisymmetric, and non-static spacetime. Thus, we neglect all other terms involving higher order than $a$ in all other quantities of interest: $\bar{R} \simeq 0, \bar{R}_{\mu \nu} \simeq$ $0, \bar{R}_{\mu \nu \rho \sigma} \neq 0, \cdots$. Importantly, the (outer) horizon is given by the Schwarzschild radius as

$$
r_{+}=2 M
$$

but the inner horizon is absent. This implies that the slowly rotating black hole has arisen from breaking spherical symmetry to axial symmetry. Up to $\mathcal{O}(a)$-order, the CS term is given by

$$
{ }^{*} \bar{R} \bar{R}(a) \simeq \frac{288 M^{2} a \cos \theta}{r^{7}}
$$

We wish to mention that Eq. (11) is a linear term which approaches zero as $a \rightarrow 0$. Considering Eq. (11), the time evolution of a spherical mode is determined mainly by 
$a \cos \theta$, in addition to the coupling parameter $\alpha$. This means that the rotation $a$ and coupling $\alpha$ play crucial roles in determining the instability bound of slowly rotating black holes, suggesting $\alpha>\alpha_{\mathrm{th}}(a)$-bound.

\section{$3 \quad$ Instability of slowly rotating black holes}

To observe the onset of slowly rotating spontaneous scalarization, we introduce the perturbations $\left(h_{\mu \nu}, \delta \phi\right)$ around the slowly rotating black hole background as

$$
g_{\mu \nu}=\bar{g}_{\mu \nu}+h_{\mu \nu}, \quad \phi=\bar{\phi}+\delta \phi \quad \text { with } \bar{\phi}=0 .
$$

The linearized equation to (3) takes a simple form like the general relativity as

$$
\delta R_{\mu \nu}(h) \simeq 0
$$

where the linearized Ricci tensor is

$$
\delta R_{\mu \nu}(h)=\frac{1}{2}\left(\bar{\nabla}^{\gamma} \bar{\nabla}_{\mu} h_{\nu \gamma}+\bar{\nabla}^{\gamma} \bar{\nabla}_{\nu} h_{\mu \gamma}-\bar{\nabla}^{2} h_{\mu \nu}-\bar{\nabla}_{\mu} \bar{\nabla}_{\nu} h\right) .
$$

The linearized scalar equation around the slowly rotating black hole takes the form

$$
\left(\bar{\nabla}^{2}-\mu_{\mathrm{CS}}^{2}\right) \delta \phi=0
$$

where an effective mass for scalar perturbation is given by

$$
\mu_{\mathrm{CS}}^{2}=-2 \alpha^{*} \bar{R} \bar{R}(a)
$$

We note that a tensor-stability analysis for the slowly rotating black hole with Eq. (13) is the same as in general relativity, implying that there are no unstable tensor modes around the slowly rotating black hole background [16]. Accordingly, the instability of slowly rotating black hole will be determined solely by the linearized scalar equation (15) in the EsCS theory. For Kerr black hole found from the EsCS theory, it was shown that the situation with negative $\alpha$ is the same as the case with positive $\alpha$ [9] because Eq. (15) is invariant under the transformation of $\alpha \rightarrow-\alpha$ and $\theta \rightarrow \pi-\theta$ [12]. Initially, we wish to consider two cases of $\alpha>0$ and $\alpha<0$ separately. 

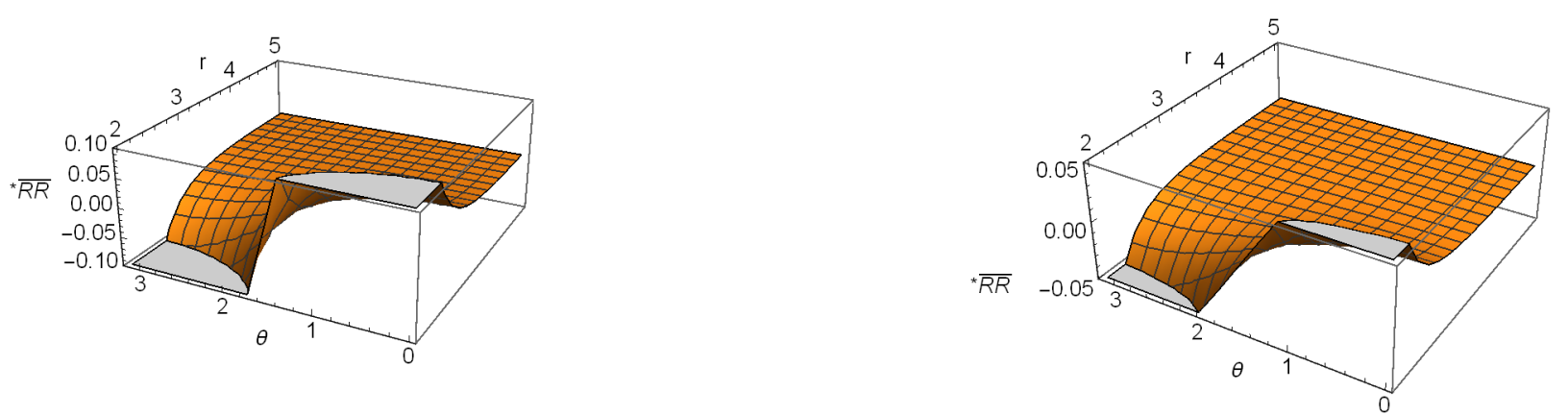

Figure 1: 3D graphs for CS term ${ }^{*} \bar{R} \bar{R}(a)$ with $M=1$ and $a=0.25$ (Left), 0.05 (Right). These include $r \in\left[r_{+}=2,5\right]$ and $\theta \in[0, \pi]$. Two graphs show division of $(+/-)$-regions near the horizon, implying no distinction in $\mu_{\mathrm{CS}}^{2}$ between positive and negative $\alpha$ when combining with the transformation of $\alpha \rightarrow-\alpha$ and $\theta \rightarrow \pi-\theta$.

\section{$3.1 \alpha>0$ case}

We find that ${ }^{*} \bar{R} \bar{R}(a)$ is an odd function with respect to $\cos \theta$. One finds the division of $(+)$-region $=(-)$-region near the horizon for any $a>0$ (See Fig. 1), implying that it could not distinguish positive coupling from negative coupling [10, 9]. We remind the reader that the threshold curve $\alpha=\alpha_{\mathrm{th}}(a)$ indicating the boundary between stable and unstable black holes depends on $a$. The threshold curve will be determined by carrying out numerical simulations. We will take a long time to obtain it.

Let us briefly explain the $(2+1)$-dimensional hyperboloidal foliation method to solve Eq. (15) numerically [10]. Firstly, we introduce the ingoing Kerr-Schild coordinates $\{\tilde{t}, r, \theta, \tilde{\varphi}\}$ by considering the coordinate transformations

$$
d \tilde{t}=d t+\frac{2 M r}{\Delta} d r, \quad d \tilde{\varphi}=d \varphi+\frac{a}{\Delta} d r .
$$

In this case, the linearized scalar equation (15) could be rewritten compactly as

$$
\begin{aligned}
\left(\rho^{2}+2 M r\right) \partial_{\tilde{t}}^{2} \delta \phi & =2 M \partial_{\tilde{t}} \delta \phi+4 M r \partial_{\tilde{t}} \partial_{r} \delta \phi+\partial_{r}\left(\Delta \partial_{r} \delta \phi\right)+2 a \partial_{r} \partial_{\tilde{\varphi}} \delta \phi \\
& +\frac{1}{\sin ^{2} \theta} \partial_{\tilde{\varphi}}^{2} \delta \phi+\frac{1}{\sin \theta} \partial_{\theta}\left(\sin \theta \partial_{\theta} \delta \phi\right)-\mu_{\mathrm{CS}}^{2} \rho^{2} \delta \phi
\end{aligned}
$$

Considering separation of variables

$$
\delta \phi(\tilde{t}, r, \theta, \tilde{\varphi})=\frac{1}{r} \sum_{m} u_{m}(\tilde{t}, r, \theta) e^{i m \tilde{\varphi}},
$$


Eq.(18) leads to a $(2+1)$-dimensional Teukolsky equation for a perturbed scalar as

$$
A^{\tilde{t} \tilde{t}} \partial_{\tilde{t}}^{2} u_{m}+A^{\tilde{t r}} \partial_{\tilde{t}} \partial_{r} u_{m}+A^{r r} \partial_{r}^{2} u_{m}+A^{\theta \theta} \partial_{\theta}^{2} u_{m}+B^{\tilde{t}} \partial_{\tilde{t}} u_{m}+B^{r} \partial_{r} u_{m}+B^{\theta} \partial_{\theta} u_{m}+C u_{m}=0,
$$

with coefficients

$$
\begin{aligned}
& A^{\tilde{t} \tilde{t}}=\rho^{2}+2 M r, \quad A^{\tilde{t} r}=-4 M r, \quad A^{r r}=-\Delta, \quad A^{\theta \theta}=-1, \\
& B^{\tilde{t}}=2 M, \quad B^{r}=\frac{2}{r}\left(a^{2}-M r\right)-2 i m a, \quad B^{\theta}=-\cot \theta, \\
& C=\frac{m^{2}}{\sin ^{2} \theta}-\frac{2\left(a^{2}-M r\right)}{r^{2}}+\frac{2 i m a}{r}+\mu_{\mathrm{CS}}^{2} \rho^{2} .
\end{aligned}
$$

As the second step, we wish to solve Eq. (21) by adopting the hyperboloidal foliation method [17] with compactified horizon-penetrating hyperboloidal (HH) coordinates $\{\tau, \rho, \theta, \tilde{\varphi}\}$ through $\tilde{t}=\tau+h(\rho)$ and $r=\rho / \Omega(\rho)$. Here, $h(\rho)=\rho / \Omega-\rho-4 M \ln \Omega$ and $\Omega=1-\rho / S$ where $S$ a free parameter determining both the domain and the foliation. This implies $\partial_{\tilde{t}}=\partial_{\tau}$ and $\partial_{r}=-\frac{d h}{d r} \partial_{\tau}+\frac{d \rho}{d r} \partial_{\rho}$. The domain $r \in\left[r_{+}, \infty\right)$ is mapped into a finite region $\rho \in\left[\rho_{+}, S\right)$ with $\rho_{+}=\left(a^{2} S+r_{+} S^{2}\right) /\left(a^{2}+2 M S+S^{2}\right)$. In this case, Eq. (21) could be written as

$$
\partial_{\tau}^{2} u_{m}=\tilde{A}^{\tau \rho} \partial_{\tau} \partial_{\rho} u_{m}+\tilde{A}^{\rho \rho} \partial_{\rho}^{2} u_{m}+\tilde{A}^{\theta \theta} \partial_{\theta}^{2} u_{m}+\tilde{B}^{\tau} \partial_{\tau} u_{m}+\tilde{B}^{\rho} \partial_{\rho} u_{m}+\tilde{B}^{\theta} \partial_{\theta} u_{m}+\tilde{C} u_{m}=0,
$$

where all coefficients appeared in [6]. Introducing a momentum $\Pi_{m}=\partial_{\tau} u_{m}$, one finds two coupled first-order equations as

$$
\begin{aligned}
& \partial_{\tau} u_{m}=\Pi_{m} \\
& \partial_{\tau} \Pi_{m}=\tilde{B}^{\tau} \Pi_{m}+\tilde{A}^{\tau \rho} \partial_{\rho} \Pi_{m}+\tilde{A}^{\rho \rho} \partial_{\rho}^{2} u_{m}+\tilde{A}^{\theta \theta} \partial_{\theta}^{2} u_{m}+\tilde{B}^{\rho} \partial_{\rho} u_{m}+\tilde{B}^{\theta} \partial_{\theta} u_{m}+\tilde{C} u_{m}
\end{aligned}
$$

The differential equations for $\rho$ and $\theta$ are solved by using the finite difference method and the time $(\tau)$ evolution is obtained by adopting the fourth-order Runge-Kutta integrator. Using the $\mathrm{HH}$ coordinates leads to the fact that the ingoing (outgoing) boundary conditions at the horizon (infinity) are satisfied automatically. On the other hand, the boundary conditions at the poles are given as $\left.u_{m}\right|_{\theta=0, \pi}=0$ for odd $m= \pm 1, \pm 3, \cdots$ and $\left.\partial_{\theta} u_{m}\right|_{\theta=0, \pi}=0$ for even $m=0, \pm 2, \cdots$.

Let us introduce a Gaussian function $\left[u_{l m}(\tau=0, \rho, \theta) \sim Y_{l m}(\theta) e^{-\frac{\left(\rho-\rho_{c}\right)^{2}}{2 \sigma^{2}}}\right]$ localized at $\rho=$ $\rho_{c}$ outside the horizon as an initial data for a perturbed scalar mode. Here $Y_{l m}(\theta)$ denotes the $\theta$-dependent part of spherical harmonics and $\sigma$ represents the standard deviation. In 


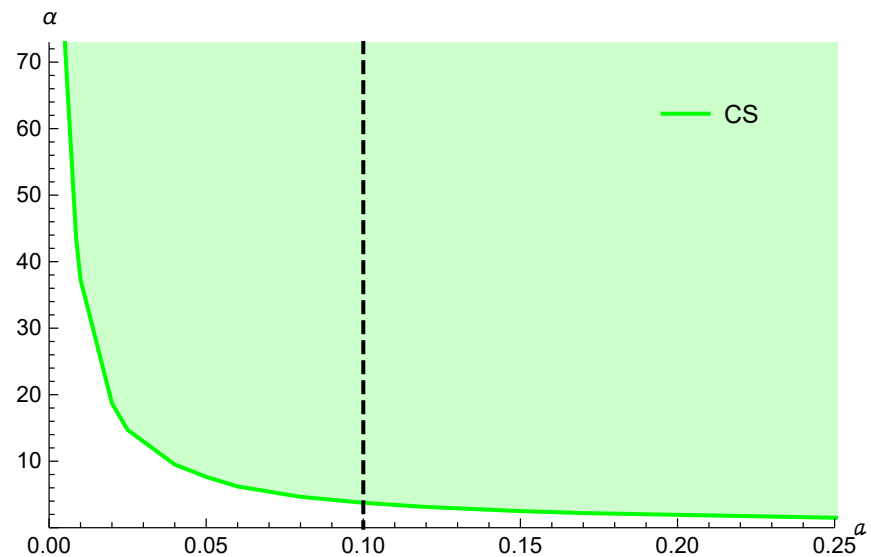

Figure 2: CS-threshold (existence) curve $\alpha=\alpha_{\text {th }}(a)$ being the boundary between stable and unstable slowly rotating black holes is obtained from observing time evolution of a spherically symmetric scalar mode for positive $\alpha$. The dashed line denotes the upper limit for fairly slow rotating black holes $(0<a \leq 0.1)$.

addition, we impose the time symmetry such that $\Pi_{l m}(\tau=0, \rho, \theta)=0$. Since the slowly rotating spacetime is axisymmetric, the mode coupling may occur.

In this work, we confine ourselves to a spherically symmetric mode of $l=m=0$ for simplicity. The time evolution for $\log _{10}\left|u_{00}(\tau, a, \alpha)\right|$ provides stable $(\searrow)$, threshold $(\longrightarrow)$, and unstable $(\nearrow)$ case with increasing time $(\tau)$. From Fig. 2, we find a threshold (existence) curve $\alpha=\alpha_{\mathrm{th}}(a)$ which indicates the boundary between stable and unstable regions based on the time evolutions of a scalar mode $\log _{10}\left|u_{00}(\tau, a, \alpha)\right| \sim \longrightarrow$. We observe that the CSthreshold curve decreases as $a$ increases and it never hits the $\alpha$-axis in the non-rotation limit $(a \rightarrow 0)$. This curve is the nearly same as in the curve obtained with Eq. (8) [9], but one might terminate around $a=0.25$ because of the slow rotation approximation. The region for fairly slow rotating black holes is given by $0<a \leq 0.1$ and the upper limit is represented by a dashed line at $a=0.1$. The unshaded region $\left[\alpha<\alpha_{\text {th }}(a)\right.$ : no growing mode $\left.(\searrow)\right]$ represents stable slowly rotating black holes, while the shaded region $\left[\alpha \geq \alpha_{\mathrm{th}}(a)\right.$ : growing mode $(\nearrow)$ ] denotes the unstable slowly rotating black holes.

\section{$3.2 \alpha<0$ case}

In this case, ${ }^{*} \bar{R} \bar{R}(a)$ and $\mu_{\mathrm{CS}}^{2}(a)$ have the same sign. The whole region near the horizon is always divided into positive $(+)$-and negative $(-)$-regions because of $\cos \theta$ in the numerator 


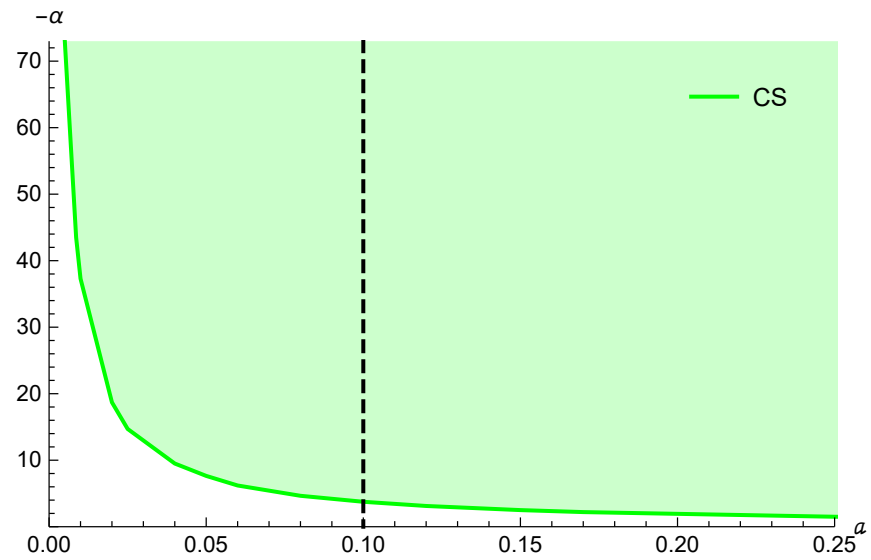

Figure 3: CS-threshold (existence) curve $\alpha=\alpha_{\text {th }}(a)$ obtained from observing the time evolution of $l=m=0$-scalar mode for negative $\alpha$. The dashed line represents the upper limit for fairly slow rotating black holes.

(see Fig. 1), implying no $a$-bound. The time evolution for $\log _{10}\left|u_{00}(\tau, a,-\alpha)\right|$ implies stable $(\searrow)$, threshold $(\longrightarrow)$, and unstable $(\nearrow)$ with increasing time $(\tau)$. From Fig. 3, we find a threshold (existence) curve $\alpha=\alpha_{\text {th }}(a)$ which is the boundary between stable and unstable regions based on the time evolutions of a scalar mode $\log _{10}\left|u_{00}(\tau, a,-\alpha)\right| \sim \longrightarrow$. Considering the invariance of Eq.(15) under the transformation of $\alpha \rightarrow-\alpha$ and $\theta \rightarrow \pi-\theta$, it is obvious that the CS-threshold curve (Fig. 3) for tachyonic instability is the same as in Fig. 2 [9]. We observe that the CS-threshold curve decreases as $a$ increases and it never hits the $-\alpha$-axis in the non-rotation limit $(a \rightarrow 0)$. The unshaded region $\left(\alpha<\alpha_{\mathrm{th}}(a)\right.$ : no growing mode) represents stable slowly rotating black holes, while the shaded region ( $\alpha \geq \alpha_{\text {th }}(a)$ : growing mode) denotes the unstable slowly rotating black holes.

\section{Discussions}

We have investigated spontaneous scalarization of slowly rotating black holes in the EsCS theory. The fairly slow rotating black holes imply the cases with $0<a \leq 0.1$ [13]. In the slow rotation approximation with $a \ll 1$, the CS term takes a linear term of $a$-rotation parameter, determining tachyonic instability. The tachyonic instability for slowly rotating black holes represents the onset of spontaneous scalarization.

We have used the $(2+1)$-dimensional hyperboloidal foliation method to show the tachy- 
onic instability of slowly rotating black holes when considering a spherically symmetric scalar-mode propagation. The time evolution for $\log _{10}\left|u_{00}(\tau, a, \alpha)\right|$ implies stable $(\searrow)$, threshold $(\longrightarrow)$, and unstable case $(\nearrow)$ with increasing time $\tau$.

It is shown that slowly rotating black holes are unstable against a spherically symmetric scalar-mode of $l=m=0$ for positive coupling $\alpha$ (Fig. 2). Taking into account the invariance of Eq.(15) under the transformation of $\alpha \rightarrow-\alpha$ and $\theta \rightarrow \pi-\theta$, the CS-threshold curve (Fig. 3) for negative coupling $\alpha$ is the same as that in Fig. 2 [9]. This means that the slowly rotating black holes are unstable for negative coupling $\alpha$ without any $a$-bound. Finally, it is not difficult to confirm the existence of 'no $a$-bound' (like $a>0$ ) by choosing the $l \rightarrow \infty$-scalar mode in the Hod's analytic approach [4, 9].

\section{Acknowledgments}

This work was supported by the National Research Foundation of Korea (NRF) grant funded by the Korea government (MOE) (No. NRF-2017R1A2B4002057). 


\section{References}

[1] P. V. P. Cunha, C. A. R. Herdeiro and E. Radu, Phys. Rev. Lett. 123, no. 1, 011101 (2019) doi:10.1103/PhysRevLett.123.011101 [arXiv:1904.09997 [gr-qc]].

[2] L. G. Collodel, B. Kleihaus, J. Kunz and E. Berti, Class. Quant. Grav. 37, no. 7, 075018 (2020) doi:10.1088/1361-6382/ab74f9 [arXiv:1912.05382 [gr-qc]].

[3] A. Dima, E. Barausse, N. Franchini and T. P. Sotiriou, Phys. Rev. Lett. 125, no. 23, 231101 (2020) doi:10.1103/PhysRevLett.125.231101 [arXiv:2006.03095 [gr-qc]].

[4] S. Hod, Phys. Rev. D 102, no. 8, 084060 (2020) doi:10.1103/PhysRevD.102.084060 arXiv:2006.09399 [gr-qc]].

[5] D. D. Doneva, L. G. Collodel, C. J. Krüger and S. S. Yazadjiev, Phys. Rev. D 102, no. 10, 104027 (2020) doi:10.1103/PhysRevD.102.104027 [arXiv:2008.07391 [gr-qc]].

[6] S. J. Zhang, B. Wang, A. Wang and J. F. Saavedra, Phys. Rev. D 102, no. 12, 124056 (2020) doi:10.1103/PhysRevD.102.124056 [arXiv:2010.05092 [gr-qc]].

[7] C. A. R. Herdeiro, E. Radu, H. O. Silva, T. P. Sotiriou and N. Yunes, Phys. Rev. Lett. 126, no. 1, 011103 (2021) doi:10.1103/PhysRevLett.126.011103 arXiv:2009.03904 [gr$\mathrm{qc}]]$.

[8] E. Berti, L. G. Collodel, B. Kleihaus and J. Kunz, Phys. Rev. Lett. 126, no. 1, 011104 (2021) doi:10.1103/PhysRevLett.126.011104 [arXiv:2009.03905 [gr-qc]].

[9] Y. S. Myung and D. C. Zou, Phys. Lett. B 814, 136081 (2021) doi:10.1016/j.physletb.2021.136081 arXiv:2012.02375 [gr-qc]].

[10] Y. X. Gao, Y. Huang and D. J. Liu, Phys. Rev. D 99, no. 4, 044020 (2019) doi:10.1103/PhysRevD.99.044020 [arXiv:1808.01433 [gr-qc]].

[11] D. D. Doneva and S. S. Yazadjiev, arXiv:2102.03940 [gr-qc].

[12] S. J. Zhang, arXiv:2102.10479 [gr-qc].

[13] J. Fuller and L. Ma, Astrophys. J. Lett. 881, no. 1, L1 (2019) doi:10.3847/20418213/ab339b [arXiv:1907.03714 [astro-ph.SR]]. 
[14] J. Lense and H. Thirring, Phys. Z. 19, 156 (1918).

[15] C. Lämmerzahl, M. Maceda and A. Macías, Class. Quant. Grav. 36, no. 1, 015001 (2019) doi:10.1088/1361-6382/aaeca7 arXiv:1802.03766 [gr-qc]].

[16] D. Hafner, P. Hintz and A. Vasy, arXiv:1906.00860 [math.AP].

[17] I. Racz and G. Z. Toth, Class. Quant. Grav. 28, 195003 (2011) doi:10.1088/02649381/28/19/195003 [arXiv:1104.4199 [gr-qc]]. 